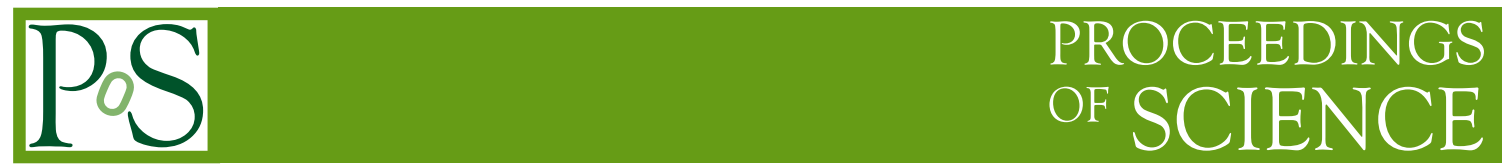

\title{
Measurements of the CKM angle $\gamma$ at BABAR
}

\author{
Fernando Martínez-Vidal ${ }^{* \dagger}$ \\ Instituto de Física Corpuscular (IFIC), Universitat de València-CSIC, \\ Apartado de Correos 22085, E-46071 Valencia, Spain \\ E-mail: fernando.martinez@ific.uv.es
}

The recent measurements of the CKM angle $\gamma$ by the BABAR experiment are reported. The analyses have been performed using the complete sample of 468 million $B \bar{B}$ pairs collected by the $B A B A R$ detector at the SLAC PEP-II asymmetric-energy $B$ factory during the years 1999-2007.

35th International Conference of High Energy Physics - ICHEP2010,

July 22-28, 2010

Paris France

* Speaker.

On behalf of the BABAR Collaboration. 
In the standard model (SM) of particle physics, $C P$ violation in the quark sector of weak interactions arises from a single irreducible phase in the Cabibbo-Kobayashi-Maskawa (CKM) that describes the mixing of quarks [1]. The unitarity of the CKM matrix $V$ defines a unitarity triangle (UT) in the complex plane. $C P$ violation measurements and semileptonic decay rates (and other methods) can be conveniently displayed and compared as constraints on the angles and sides, respectively, of this triangle. Inconsistencies between all these (in general) precise and redundant constraints can be used to search for new physics (NP). As today, there is an impressive overall agreement between all measurements [2]. Among these the angle $\gamma$, defined as the phase of $V_{u b}$ in the Wolfenstein parametrization [1], is particularly relevant since it is the only $C P$-violating measurement that, together with the determination of the $C P$-conserving magnitude of $V_{u b}$, selects a region of the UT apex independently of most types of NP, and thus constitutes a SM candle type of measurement. Current constraints, provided by the BABAR and Belle experiments, make use of $B^{ \pm} \rightarrow D^{(*)} K^{ \pm}$and $B^{ \pm} \rightarrow D K^{* \pm}$ decays, and are still weak $\left(\sim 15^{\circ}\right)$. Neutral $B$ decays have also been proposed, although do not yet provide significant constraints.

The angle $\gamma$ from $B^{ \pm} \rightarrow D^{(*)} K^{ \pm}$and $B^{ \pm} \rightarrow D K^{* \pm}$ decays is determined measuring the interference between the amplitudes $b \rightarrow u$ and $b \rightarrow c$, when the neutral $D$ meson is reconstructed in a final state accessible from both $D^{0}$ and $\bar{D}^{0}$ decays. Since both amplitudes are tree level, the interference is unaffected by NP appearing in the loops, making the theoretical interpretation of observables in terms of $\gamma$ very clean. The disadvantage is that the branching fractions of the involved decays are small due to CKM suppression $\left(10^{-5}-10^{-7}\right)$, and the size of the interference, given by the ratio $r_{B}$ between the magnitudes of the $b \rightarrow u$ and $b \rightarrow c$ amplitudes, is small due to further CKM and color suppressions $(\sim 10 \%)$. As a consequence, the measurements are statistically limited and one has to combine complementary methods applied on the same $B$ decay modes sharing the hadronic parameters ( $r_{B}$ and $\delta_{B}$, i.e. the relative magnitude and phase of the $b \rightarrow u$ and $b \rightarrow u$ transitions) and $\gamma$, and use as many as possible different $B$ decay modes to improve the overall sensitivity to $\gamma$.

In this talk we present the most recent determinations of $\gamma$ obtained by BABAR, based on the full data sample of charged $B$ meson decays produced in $e^{+} e^{-} \rightarrow \Upsilon(4 S) \rightarrow B^{+} B^{-}$and recorded in the years 1999-2007, about $468 \times 10^{6} B^{+} B^{-}$pairs. We have studied $B^{ \pm} \rightarrow D^{(*)} K^{ \pm}$and $B^{ \pm} \rightarrow D K^{* \pm}$ decays, with the neutral $D$ mesons reconstructed in a number of different final states: $D \rightarrow K_{S}^{0} h^{+} h^{-}$, with $h=\pi, K$ (Dalitz plot method); $D \rightarrow K^{ \pm} \pi^{\mp}$ (ADS method); and $D \rightarrow f_{C P}$, with $f_{C P}$ a $C P$ eigenstate (GLW method) [3].

One of the charged $B$ mesons produced in the $\Upsilon(4 S)$ decay is fully reconstructed, with efficiencies ranging between $40 \%$ (for low-multiplicity decays with no neutrals) and $10 \%$ (for highmultiplicity decays with neutrals). The selection is optimized to maximize the statistical sensitivity. The reconstruction efficiencies have substantially improved (20\% to $60 \%$ relative) with respect to our previous measurements based on $384 \times 10^{6} B^{+} B^{-}$pairs, reflecting improvements in tracking and particle identification, and optimization of analysis procedures. Signal $B$ decays are characterized by means of two nearly independent kinematic variables exploiting the constraint from the known beam energies: the beam-energy $m_{\mathrm{ES}} \equiv \sqrt{E_{\text {beam }}^{* 2}-\left|p_{B}^{*}\right|^{2}}$ and the energy-difference $\Delta E \equiv E_{B}^{*}-E_{\text {beam }}^{*}$. Since the main source of background comes from $q \bar{q}$ continuum production, additional discrimination is achieved using multivariate analysis tools, from the combination (either a linear Fisher discriminant $\mathscr{F}$, or a non-linear neural network $N N$ ) of several event-shape quanti- 
ties. These variables distinguish between spherical $B \bar{B}$ events from more jet-like continuum events and exploit the different angular correlations in the two event categories. The signal is finally separated from background through unbinned maximum likelihood (UML) fits to the $B^{ \pm} \rightarrow D^{(*)} K^{ \pm}$ and $B^{ \pm} \rightarrow D K^{* \pm}$ data using $m_{\mathrm{ES}}, \Delta E$, and $\mathscr{F}$ or $N N$. $B^{ \pm} \rightarrow D^{(*)} \pi^{ \pm}$decays, which are about 12 times more abundant than $B^{ \pm} \rightarrow D^{(*)} K^{ \pm}$, have a similar topology but are discriminated by means of excellent pion and kaon identification provided by $d E / d x$ and Cerenkov measurements, and show negligible $C P$-violating effects $\left(r_{B} \sim 1 \%\right)$. Therefere, these decays provide powerful calibration and control samples for negative tests of $C P$ violation.

In the Dalitz plot (DP) method the amplitude for a $B^{-}$decay has for the $b \rightarrow c$ transition the DP of the $D^{0}$ decay, while for the $b \rightarrow u$ transition the DP is the corresponding to the $\bar{D}^{0}$ decay. If we assume no $D$ mixing nor $C P$ violation in the $D$ decay, and use as independent kinematic variables $s_{ \pm}=m^{2}\left(K_{s}^{0} \pi^{ \pm}\right)$, then the two DPs are one rotated $90^{\circ}$ to each other. This is of critical importance since allows to determine directly from data the strong charm phase variation for $D^{0}$ and $\bar{D}^{0}$, as well as well as the hadronic parameters $r_{B}$ and $\delta_{B}$, and the weak phase $\gamma$, provided that a $D$ decay amplitude model is assumed. For $B^{+}$decays one has to interchange the $D^{0}$ and $\bar{D}^{0}$ DPs, and change the sign of $\gamma$. This results in an interference term proportional to our observables $x_{ \pm} \equiv r_{B} \cos \left(\delta_{B} \pm \gamma\right)$ and $y_{ \pm} \equiv r_{B} \sin \left(\delta_{B} \pm \gamma\right)$, i.e. the real and imaginary parts of the ratio of $b \rightarrow u$ and $b \rightarrow c$ amplitudes for $B^{ \pm}$decays. We reconstruct $B^{ \pm} \rightarrow D K^{ \pm}, D^{*} K^{ \pm}$with $D^{*} \rightarrow D \pi^{0}, D \gamma$, and $B^{ \pm} \rightarrow D K^{* \pm}$ with $K^{* \pm} \rightarrow K_{s}^{0} \pi^{ \pm}$decays, followed by neutral $D$ meson decays to the 3-body self-conjugate final states $K_{S}^{0} h^{+} h^{-}$, with $h=\pi, K$. From the UML fit we determine the signal and background yields in each of the eight different final states for each $B$ charge, along with the $C P$ violating parameters $x_{ \pm}$and $y_{ \pm}$[4]. We find $1507 B^{ \pm}$signal candidates with $K_{S}^{0} \pi^{+} \pi^{-}$, and 268 with $K_{S}^{0} K^{+} K^{-}$. Prior to the $C P$ fit, we model the $D^{0}$ and $\bar{D}^{0}$ decay amplitudes as a coherent sum of S-, $\mathrm{P}-$, and D-waves, and determine their amplitudes and phases (along with other relevant parameters) relative to the dominant two-body $C P$-eigenstates $K_{S}^{0} \rho(770)$ (for $K_{S}^{0} \pi^{+} \pi^{-}$) and $K_{S}^{0} a_{0}(980)$ (for $\left.K_{S}^{0} K^{+} K^{-}\right)$, using a large $\left(\approx 6.2 \times 10^{5}\right)$ and very pure $(\approx 99 \%)$ signal sample of flavor tagged neutral $D$ mesons from $D^{*+} \rightarrow D^{0} \pi^{+}$decays produced in $e^{+} e^{-} \rightarrow c \bar{c}$ events [5]. From the $\left(x_{ \pm}, y_{ \pm}\right)$
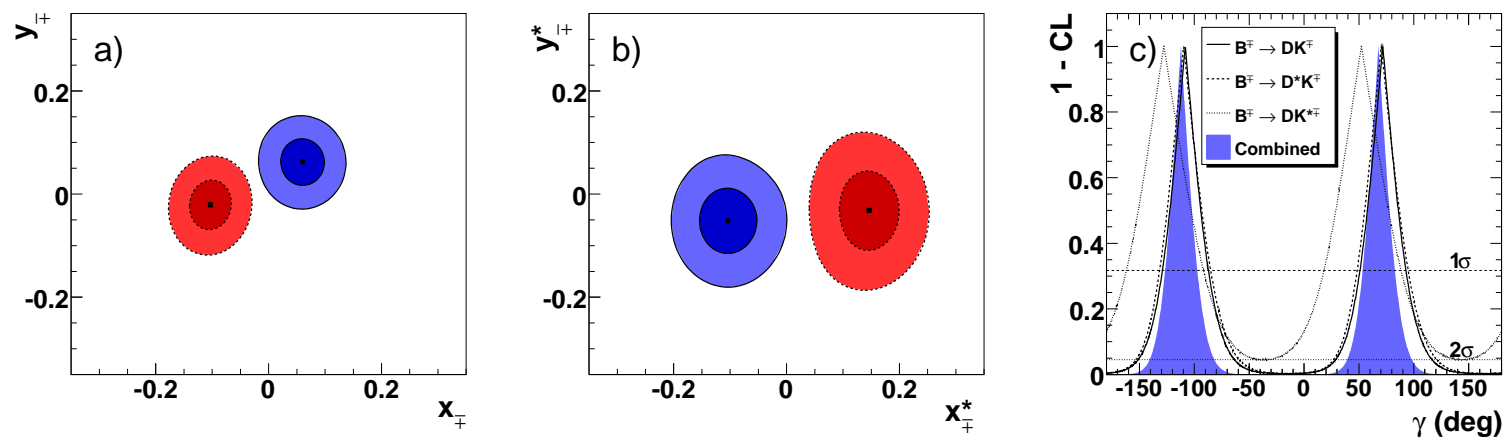

Figure 1: $1 \sigma$ and $2 \sigma$ contours in the $\left(x_{ \pm}, y_{ \pm}\right.$) planes for (a) $B^{ \pm} \rightarrow D K^{ \pm}$and (b) $B^{ \pm} \rightarrow D^{*} K^{ \pm}$, for $B^{-}$(solid lines) and $B^{+}$(dotted lines) decays. (c) $1-\mathrm{CL}$ as a function of $\gamma$ for $B^{ \pm} \rightarrow D K^{ \pm}, D^{*} K^{ \pm}, D K^{* \pm}$ decays. The dashed (upper) and dotted (lower) horizontal lines correspond to the $1 \sigma$ and $2 \sigma$ intervals, respectively.

confidence regions for each of the 3 different $B$ decay modes -Fig. 1.(a)(b)- we determine, using a frequentist procedure, $1 \sigma[2 \sigma]$ intervals for $\gamma$-Fig. 1.(c)-. We obtain $\gamma\left(\bmod 180^{\circ}\right)=(68 \pm$ 
$14 \pm 4 \pm 3)^{\circ}\left[39^{\circ}, 98^{\circ}\right]$, where the three uncertainties are statistical, experimental systematic, and amplitude model systematic. We also determine the hadronic parameters $r_{B}^{D K^{ \pm}}=(9.6 \pm 2.9) \%$ $[3.7,15.5] \%, r_{B}^{D^{*} K^{ \pm}}=\left(13.3_{-3.9}^{+4.2}\right) \%[4.9,21.5] \%, \kappa r_{B}^{D K^{* \pm}}=\left(14.9_{-6.2}^{+6.6}\right) \%[0,28.0] \%(\kappa=0.9 \pm 0.1$ takes into account the $K^{*}$ intrinsic width), and the strong phases $\delta_{B}^{D K^{ \pm}}, \delta_{B}^{D^{*} K^{ \pm}}$, and $\delta_{B}^{D K^{* \pm}}$ [4]. A $3.5 \sigma$ evidence of direct $C P$ violation $(\gamma \neq 0)$ is found from the combination of the 3 channels, which corresponds to the significance of the separation between the $\left(x_{+}, y_{+}\right)$and $\left(x_{-}, y_{-}\right)$solutions in Fig. 1.(a)(b).

In the ADS method, we reconstruct $B^{ \pm} \rightarrow D K^{ \pm}, D^{*} K^{ \pm}$with $D^{*} \rightarrow D \pi^{0}, D \gamma$, followed by $D$ decays to both the doubly-Cabibbo-suppressed (DCS) $D^{0}$ final state $K^{+} \pi^{-}$and the Cabibbofavored (CF) $K^{-} \pi^{+}$, which is used as normalization and control sample. Final states with oppositesign kaons arise either from the CKM favored $B$ decay followed by the DCS $D$ decay or from the CKM- and color-suppressed $B$ decay followed by the CF $D$ decay, producing an interference which can be potentially large since the magnitudes of the interfering amplitudes are similar. However, their overall branching ratios are very small $\left(\sim 10^{-7}\right)$ and background suppression becomes crucial. The UML fit directly determines the three branching fraction ratios $R_{A D S}$ between $B$ decays with opposite-sign and same-sign kaons, and the three yields of $B$ decays with same-sign kaons, using $m_{\mathrm{ES}}$ and $N N$. The three $C P$ asymmetries $A_{A D S}$ are inferred from all these. We obtain first indications of signals for the $B^{ \pm} \rightarrow D K^{ \pm}$and $B^{ \pm} \rightarrow D^{*} K^{ \pm}$(with $D^{*} \rightarrow D \pi^{0}$ ) opposite-sign modes -Fig. 2-, with significances of $2.1 \sigma$ and $2.2 \sigma$, respectively [6]. The measured branching fraction ratios are $R_{A D S}^{D K}=(1.1 \pm 0.5 \pm 0.2) \times 10^{-2}, R_{A D S}^{\left[D \pi^{0}\right] K}=(1.8 \pm 0.9 \pm 0.4) \times 10^{-2}$, and $R_{A D S}^{[D \gamma] K}=(1.3 \pm 1.4 \pm 0.8) \times 10^{-2}$, and the $C P$ asymmetries are $A_{A D S}^{D K}=-0.86 \pm 0.47_{-0.16}^{+0.12}$, $A_{A D S}^{\left[D \pi^{0}\right] K}=0.77 \pm 0.35 \pm 0.12$, and $A_{A D S}^{[D \gamma] K}=0.36 \pm 0.94_{-0.41}^{+0.25}$. From these results and external measurements of the relative amplitude and phase of $\bar{D}^{0}$ to $D^{0}$ mesons decaying into the $K^{-} \pi^{+}$ final state [7] we infer, using a frequentist procedure similar to that used in the DP method, $r_{B}^{D K^{ \pm}}=\left(9.5_{-4.1}^{+5.1}\right) \%[0,16.7] \%, r_{B}^{D^{*} K^{ \pm}}=\left(9.6_{-5.1}^{+3.5}\right) \%[0,15.0] \%$, and the strong phases $\delta_{B}^{D K^{ \pm}}, \delta_{B}^{D^{*} K^{ \pm}}$, in good agreement with those obtained with the DP technique.
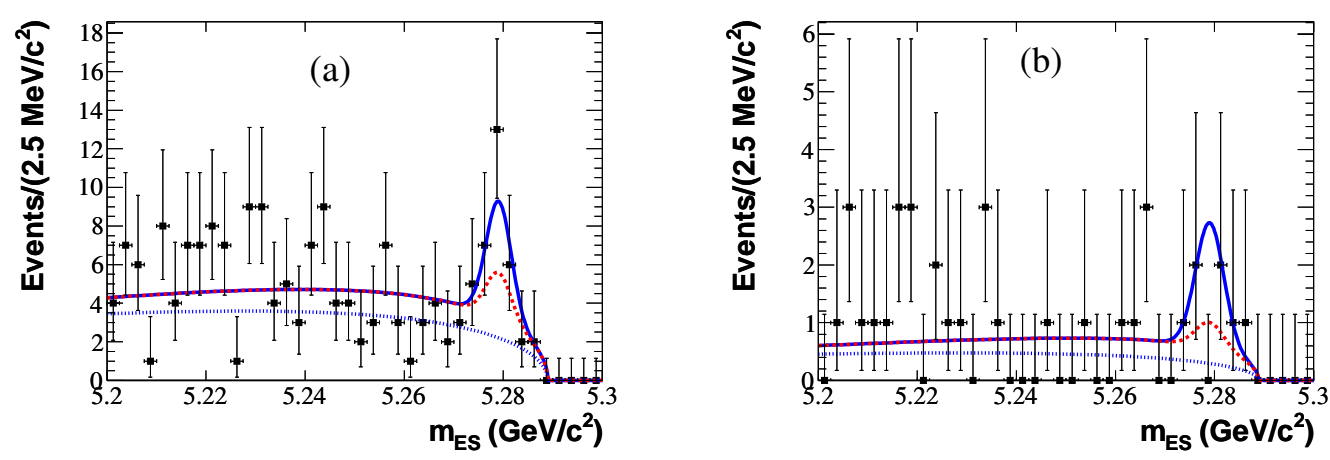

Figure 2: Projections on $m_{\mathrm{ES}}$ for (a) $B^{ \pm} \rightarrow D K^{ \pm}$and (b) $B^{ \pm} \rightarrow D^{*}\left[D \pi^{0}\right] K^{ \pm}, D \rightarrow K^{\mp} \pi^{ \pm}$opposite-sign decays, for ADS samples enriched in signal $(N N>0.94)$. The points with error bars are data while the curves represent the fit projections for signal plus background (solid), the sum of all background components (dashed), and $q \bar{q}$ background only (dotted).

In the GLW method, we reconstruct $B^{ \pm} \rightarrow D K^{ \pm}$decays, followed by $D$ decays to non- $C P$ 
$\left(D^{0} \rightarrow K^{-} \pi^{+}\right), C P$-even $\left(K^{+} K^{-}, \pi^{+} \pi^{-}\right)$, and $C P$-odd $\left(K_{S}^{0} \pi^{0}, K_{S}^{0} \phi, K_{S}^{0} \omega\right)$ eigenstates. The partial decay rate charge asymmetries $A_{C P \pm}$ for $C P$-even and $C P$-odd $D$ final states and the ratios $R_{C P \pm}$ of the charged-averaged $B$ meson partial decay rates in $C P\left(R_{K / \pi}^{ \pm}\right)$and non- $C P\left(R_{K / \pi}\right)$ decays (normalized to the corresponding $B^{ \pm} \rightarrow D \pi^{ \pm}$decays, to reduce systematic uncertainties) provide four observables from which the three unknowns $\gamma, r_{B}$ and $\delta_{B}$ can be extracted (up to an 8-fold ambiguity for the phases). The signal yields, expressed in terms of $A_{C P \pm}, R_{K / \pi}^{ \pm}$and $R_{K / \pi}$ are extracted from UML fits to $m_{\mathrm{ES}}, \Delta E$, and $\mathscr{F}$. We identify about $500 B^{ \pm} \rightarrow D K^{ \pm}$ decays with $C P$-even $D$ final states and a similar amount for $C P$-odd $D$ final states, and measure [8] $A_{C P+}=0.25 \pm 0.06 \pm 0.02, A_{C P-}=-0.09 \pm 0.07 \pm 0.02, R_{C P+}=1.18 \pm 0.09 \pm 0.05$, and $R_{C P-}=1.07 \pm 0.08 \pm 0.04$. The parameter $A_{C P+}$ is different from zero with a significance of $3.6 \sigma$, and constitutes evidence for direct $C P$ violation in $B^{ \pm} \rightarrow D K^{ \pm}$decays. These results can be written in terms of the observables $x_{ \pm}$using the relationship $x_{ \pm}=\left[R_{C P+}\left(1 \mp A_{C P+}\right)-R_{C P-}\left(1 \mp A_{C P-}\right)\right] / 4$. Excluding the $D \rightarrow K_{S}^{0} \phi, \phi \rightarrow K^{+} K^{-}$channel to facilitate the combination with the DP method, we find $x_{+}=-0.057 \pm 0.039 \pm 0.015$ and $x_{-}=0.132 \pm 0.042 \pm 0.018$, which are consistent (and of similar precision) with the DP method. From these results and using a frequentist procedure similar to that used previously we infer $24 \%<r_{B}<45 \%[6,51] \%$, and $\bmod 180^{\circ}, 11^{\circ}<\gamma<23^{\circ}$ or $81^{\circ}<\gamma<99^{\circ}$ or $157^{\circ}<\gamma<169^{\circ}\left[7^{\circ}, 173^{\circ}\right]$.

We have reported the recent progress in the determination of the CKM angle $\gamma$, using the complete BABAR data sample and three different and complementary methods (DP, ADS, and GLW). A coherent and consistent set of results on $\gamma$ and the hadronic parameters characterizing the $B$ decays has been obtained. The central value for $\gamma$, around $70^{\circ}$ with a precision around $15^{\circ}$, is consistent with indirect determinations from CKM fits [2]. A proper average of all the three methods using the full BABAR sample of $B^{ \pm} \rightarrow D^{(*)} K^{ \pm}, D K^{* \pm}$ decays is foreseen. We obtain $x_{-}-x_{+}=0.175 \pm 0.040$ by combining the $x_{ \pm}$measurements from the DP and GLW methods for $B^{ \pm} \rightarrow D K^{ \pm}$decays, which is different from zero with a significance of $4.4 \sigma$, thus constitutes strong evidence for direct $C P$ violation in these charged $B$ decays. Finally, we have the first sign of an ADS signal in $B^{ \pm} \rightarrow D K^{ \pm}$ and $B^{ \pm} \rightarrow D^{(*)} K^{ \pm}$decays.

\section{References}

[1] N. Cabibbo, Phys. Rev. Lett. 10, 531 (1963); M. Kobayashi and T. Maskawa, Prog. Theor. Phys. 49, 652 (1973); L. Wolfenstein, Phys. Rev. Lett. 51, 1945 (1983).

[2] J. Charles et al., http://ckmfitter.in2p3.fr/; M. Bona et al., http://www.utfit.org/.

[3] A. Giri, Y. Grossman, A. Soffer and J. Zupan, Phys. Rev. D 68, 054018 (2003); D. Atwood, I. Dunietz and A. Soni, Phys. Rev. Lett. 78, 3257 (1997); Phys. Rev. D 63, 036005 (2001); M. Gronau and D. London, Phys. Lett. B 253, 483 (1991); M. Gronau and D. Wyler, Phys. Lett. B 265, 172 (1991).

[4] P. del Amo Sanchez et al. (BABAR Collaboration), Phys. Rev. Lett. 105, 121801 (2010); B. Aubert et al. (BABAR Collaboration), Phys. Rev. D 78, 034023 (2008).

[5] P. del Amo Sanchez et al. (BABAR Collaboration), Phys. Rev. Lett. 105, 081803 (2010).

[6] P. del Amo Sanchez et al. (BABAR Collaboration), Phys. Rev. D 82, 072006 (2010).

[7] E. Barberio et al. (HFAG Group), arXiv:0808.1297v3.

[8] P. del Amo Sanchez et al. (BABAR Collaboration), Phys. Rev. D 82, 072004 (2010). 